\title{
The Adoption and Usages of Technology in SMEs: The Case of Making Tax Digital
}

\author{
Dimitra Skoumpopoulou ${ }^{\circledR}$, Adam Crisp \\ Northumbria University, Newcastle upon Tyne NE1 8ST, United Kingdom \\ E-mail: d.skoumpopoulou@northumbria.ac.uk
}

Received: 21 April 2020; Revised: 29 May 2020; Accepted: 29 July 2020

\begin{abstract}
Technology is a rapidly developing concept and its development is second to none while technology and the internet is more available than ever. Its uses range from personal use, up to and including large corporate organisations. However, this study will be closely looking into Small Medium Enterprises (SMEs) and their perception of technology. With SMEs taking up the largest portion of all businesses in the UK, they are the majority target audience to study. This research will into the adoption and usage of technologies by SMEs and more specifically we will explore the case of Making Tax Digital (MTD). This includes a review of the academic literature surrounding SMEs and the use of technology. We will be discussing the research methods used within this study and the research results gathered from our primary respondents. This study uses both secondary and primary research in an effort to develop further understanding of SMEs and their close relation to technology. The study investigates the future of Making Tax Digital and the affect it will have on SMEs while it summarises how businesses must adapt MTD and face the new system's requirements.
\end{abstract}

Keywords: Small and Medium Enterprises (SMEs), Making-Tax-Digital, technology perception

\section{Introduction}

Worldwide technology usage has more than doubled in the past ten years and continues to become more available worldwide [1]. Due to these developments, technology and Information Technology (IT) are gaining traction worldwide. Additionally, technology is becoming a larger part in everyday society, with the use of mobile phones and tablets. However, with the development of new technology comes additional expansion and usage of the Internet. The Internet it being used by all generations [2], and is creating opportunities for businesses to grow via international markets [3]. As the Internet continues to cultivate, the impact it has on businesses is increasing and is becoming multidimensional. New systems and technologies adoption become imperative for the success of businesses and one of the most recent developments is Making Tax Digital (MTD), a government legislation that will enable companies to transition their tax payments to an online service.

The number of Small and Medium Enterprises (SMEs) has grown by $63 \%$ since 2000, and SMEs are said to be $99 \%$ of the UK's economy [4]. Thus, this study will focus on SMEs perception of the proposed changes and developments in technology and it will aim to shed more light into understanding how SMEs can adapt to the new government policy of MTD.

Copyright (C2020 Dimitra Skoumpopoulou, et al.

DOI: https://doi.org/10.37256/est.212021382

This is an open-access article distributed under a CC BY license

(Creative Commons Attribution 4.0 International License)

https://creativecommons.org/licenses/by/4.0/ 


\section{Benefits for SMEs using technology}

In order to gain an insight into how SMEs decide whether to use new technologies, it is important to understand the main benefits and drawbacks of technologies for SMES. With the development of policies designed to facilitate innovation and technological adoption within SMEs being ever created [5], it is argued that technology is more available than ever for SMEs [2], and therefore its extensive use becomes essential for them.

Researchers [6]-[9] argue that after the initial implementation costs, IT is proven to help in both long-term cost reduction and operations' effectiveness. Similarly, Li et al. [10] found that introducing new technologies could enable businesses to improve their team building and organisational capability.

On a larger geographical scale, SMEs have access to a widespread opportunity to participate in the regional and international markets as well as to develop and expand their customer base globally [11]. Since Alam and Mohammad Noor [11] came to this conclusion, there have been vast developments in what the Internet and IT can offer. This not only gives the opportunity of expansion to SMEs, but nowadays it offers them the opportunity to source suppliers and materials from the international market, which has its own expansive list of benefits. For example, it enables businesses to find suppliers away from their local environment and this often leads to cost savings as well as the sourcing of new and different products which consequently can make them more competitive [12], [13].

Moreover, Shiels et al. [14] believe that the development of the Internet has forced businesses to review their existing practices and create new methods of operating in line with the Internet and the new digital world. More specifically, Stankovska et al. [15] argue that just adopting a new technology is not enough to source a competitive advantage. Organisations must use these advances in technology not only as a way to source information or products but they have to go a step further so that they can effectively engage and build relationships with their consumers [16], [17]. Therefore, when implementing a new IT, a company must allow themselves the time and resources so that they can ensure that the transition and adoption of the new technology goes smoothly while they have to manage unrealistic expectations of instant improvements and savings. Often adopting the wrong technology and not embracing the change that a new system brings are the biggest flaws in introducing new technologies for SMEs [18]. In light of this, the next section will aim to explore the reasons that lead SMEs to use and adopt technological change.

\section{SMEs and technological change}

The existing literature suggests that there are many reasons why SMEs adopt and use new technologies [8], [9], [11], [19]. Parthasarathy and Kumar [19] found that IT adoption within SMEs plays a vital role in helping the standardisation process, therefore they believe that without being able to adapt and adopt to changes in IT developments, this could potentially hinder SMEs performance. Since adopting a new technology would help with standardisation, SMEs cannot ignore the importance and relevance of them. Parthasarathy and Kumar [19] further argue that the adoption of IT would allow SMEs to better standardise their processes whether this is about storing data or sending important documents to clients and customers. By having a level of standardisations SMEs can be consistent towards their customers and suppliers and this further strengthens their business image and minimises the possibilities of errors and complains.

Mehrtens et al. [20] believe that external pressure and external factors influence the use of IT and argue that various factors have different levels of influence on SMEs. However, they highlight that external factors are having the largest influence on SMEs. Also according to Shiels et al. [14], there is a possibility that companies have a pre-existing perception of the use of technology nowadays because of the development of the Internet. Businesses, often feel that they have to go along with technological advancements if they want to stay in businesses and retain their customers.

Similarly, there are various reasons for SMEs deciding to start adopting new technologies and Ghobakhloo et al. [21] categorised them into internal (owner / manager characteristics, organisational behaviour characteristics, firm's resources and IT users) and external factors (IT products in market, External and competitive pressures, external IT consultant and vendors and government). Ghobakhloo et al. [21] further mention that additional influencing factors that must also be considered include; management, resources and end users. These influencing factors though are often the main limitations that SMEs face during IT adoptions due to their size, scale and financial constraints.

In addition, Celuch [22] found that there is a direct correlation between SMEs perception on technology and the 
intention of adopting new technology; the better the perception the more likely to adopt, and vice versa. This explains that SMEs are more likely to be influenced by normative social influences [22].

Baines [23] suggests that the use of IT is often more strenuous on SMEs and could damage working life and potentially home life. This was believed to be the case as at the time many people refused technological changes as they thought it was being forced upon them. From a different perspective, Quayle [6] disagreed with this view and argued that the idea of forcing IT upon SMEs is the result of poor perception and poor understanding of the benefits of new technologies. For example, Levy and Powell [24] suggest in their study that SMEs must recognise the value of the Internet alongside a positive technological attitude, and conclude that there are key factors in business growth and success. This further builds on the idea of Harindranath et al. [25] who suggest that there is a positive correlation between choice, perception and business success.

Harindranath et al. [25] found that the nature of which technology is adopted, is directly correlated to the opinions of managers, since they are the ones who make the final decisions. Thus, if managers feel they have the power to choose which technology to implement, then they respond more positively towards the new systems, while if they feel forced, they respond negatively towards it. Using Ghobakhloo et al.'s [21] research and integrating it with that of Harindranath et al. [25], it could be argued that the internal factors come from choice, whereas external factors may come from force as Harindranath et al. [25] suggest in their study. This information can be key when looking into the future of new technologies such as Making Tax Digital [26] since businesses' freedom to make a choice could be seen as a key motivator of success for this new tax system. More specifically, applying this idea to SMEs would suggest that choice might be a key factor in perception and attitude towards the implementation and adoption of MTD across the UK. Furthermore, Stankovska et al. [15] believe that when a technology is forced upon SMEs then that follows up with further negative comments / perceptions towards the new technology. Therefor choice and perceptions are closely linked and this is an area that will be investigated further in this study.

However, Dholakia and Kshetri [27] also found that those who have prior experience with technologies implementation perceive new IT adoptions in a more positive light. Also, the general IT usage in younger generations may be more accepting and positive since they are more familiar with the current technological advancements, whereas older generations could be more likely to oppose changes to technology as they are generally less IT literate.

After reviewing the literature, it seems that SMEs are very conscious of the differences between internal and external factors that influence their decisions on which technologies they have to adopt in their business. However, companies often feel that they have no clear choice but to introduce new technologies if they are to stay on top of their operations and maintain their performance. This research will explore the extent to which choice and previous experience are key factors in how SMEs perceive new technologies and will specifically consider these in the context of Making Tax Digital.

\section{The future of Making Tax Digital (MTD)}

The external factors of recent times are set to have a big focus and a fair impact upon SMEs, most recently and specifically the development of the government's initiative of Making Tax Digital (MTD). The government describes the intent of MTD is to make it easier for individuals and businesses to get their tax right, keep on top of their affairs, as well as to be more effective, efficient, and easier to get their tax right with the aim to reduce stress and unwanted errors [28].

Looking into the motivations of introducing MTD, some critics will argue that this technology has been forced onto SMEs due to external factors such as government policy makers in order to ensure greater transparency and accountability. Thus, by using the work of Ghobakhloo et al. [21] and Harindranath et al. [25], this might suggest that SMEs would perceive this new technology negatively while the requirement to implement and introduce it in their business might not be seen favourably.

Sawyer and James [29] are one of the limited studies looking into the digitalisation of tax in New Zealand and UK. They argue that there are growing concerns over the impact that this new initiative will have on businesses and they point out that possibly the biggest challenge that these businesses will face will be issues related to the technological advancements that are related to the digitalisation of tax. As the concept of MTD is new, and since there is not a large amount of existing research surrounding the topic, Sawyer and James [29] call for more research to be conducted in this 
domain. After looking at available information surrounding MTD our research responds to this call and thus, our main aim is to explore "How will SMEs adopt 'Making Tax Digital'?"

Existing literature provides lots of information surrounding how SMEs should adopt new technologies. However, the focus of this research is to make an effort to align SMEs perceptions and adoption of new technologies such as MTD. This study will link closely the perceptions that businesses have of technological advancements such as MTD and will endeavour to gain a better understanding of how SMEs really feel and perceive this new technology.

\section{Methodology}

Due to the nature of the research and since we are interested in gaining a better understanding of how SMEs adopt and use technology as well as how they perceive the new MTD technology, the authors decided on adapting a qualitative stance towards conducting our research.

After critically reviewing the literature we found that there is a gap in getting an understanding of how SMEs perceive and adapt new technologies. We identified a number of key areas to investigate according to the literature discussed above and these areas are:

1. Reasons that SMEs use technology and how technology affects their business.

2. Are SMEs deciding in using IT because of choice or force and how each of these factors shapes the way businesses use technologies.

3. SMEs perceptions of current and future developments in technology.

4. SMEs perceptions on MTD.

5. Are new technologies necessary for SMEs?

When deciding upon potential data collection methods it was crucial to refer to the key areas of interest to ensure each step taken is in line with each key area. Due to the nature of each key area being perception and opinion driven, it is clear that the study would need to be qualitative in nature [30]. Also, we followed Gibbs's [31] study, and we decided that one-to-one interviews will provide us with rich qualitative data.

Marshall [32] comments on sample size being important to qualitative research, implying that a greater number of participants might provide better quality of research. The authors undertook 10 semi-structured interviews. Gibbs [31] deems this to be enough interviews to gain an accurate response from a group of people, stating that the more interviews the more reliable the response. Using semi-structured interviews allowed greater flexibility and freedom for participants to share information they felt was important. This would in turn give a better idea of what issues were more important to each participant.

Using data analysis research conducted by Miles and Huberman [33], the authors categorised each question and response into each key area of interest. Each interview followed a list of set questions derived from each of the 5 areas of interest (as explained above), which in turn were derived from the literature review, and investigated via further questions in the format of semi-structured interviews. During each interview the authors attempted to stick to each area of interest, while also allowing interviewees to develop on additional areas they felt important.

After completing each interview, the authors' organised responses back into each area of interest and allowed comparison from the feedback among responses. From comparing feedback from each area, the authors looked for common trends among participants. From here, the authors managed to gain a greater understanding from each participant's feedback and develop further knowledge of the situation. The next section discusses the findings from the primary research and these finding are grouped according to the 5 key areas of interest discussed above.

\section{Findings and discussion}

\subsection{SMEs and the use of technology}

This first area was to find the reasons why SMEs use technology. There is a large amount of existing research explaining why IT helps SMEs; however, this area will help develop a better understanding whether SMEs realise these benefits, and whether SMEs realise how technology affects their business. 
From conducting primary research, most participants were confident that IT helped their daily activity by making it easier to control and monitor their business by having all information in one place.

One participant said "it helps organise everything into one place and saves me time". With another participant explaining "we use online banking all the time, so we get that it helps us". The general theme was consistent of helping making their lives easier and little regarding the benefits the business may have from using technology. Referring to the literature Quayle [6] shared the concept of reductions in both long-term cost and time. After looking back at the literature, the suggestion of time and cost savings appears to be clear and the primary data gives a stronger insight into how SMEs view these benefits, as they view them in a personal light rather than a business mentality.

However, a participant from a larger company gave more in-depth feedback surrounding why their company uses technology. The participant highlighted that "using technology gives our company current and up to date information", and further explaining "it (technology) also helps us adhere to regulations".

It is interesting to note that none of the participants directly mentioned that technology aids in standardisation and consistency. Parthasarathy and Kumar [19] described IT to help standardisation and stated this as a very important factor SMEs must consider. After mentioning the concept of standardisation in the interview, participants seemed to agree with the concept, however, this was no more than a simple nod in response most of the time.

Furthermore, there was no mention of access to international markets [19] in the form of buying or selling. Nor did any of these businesses consider the global influences that technology would have on SMEs. This would suggest that advertising the global market to SMEs may be of use. However, buying and selling on a regional level were mentioned. The example being it allowed the participant to check stocks of wholesalers before travelling out of their way to buy supplies.

It seems SMEs do notice the benefits of using IT, however, the benefit is generally considered to be predominantly an improvement of efficiency and self-interest.

\subsection{Reasons that shape how SMEs use technologies}

The literature review showed that the nature of the adoption, via the IT adoption for SMEs framework [21], is directly related to the idea of choice and perception toward the new technology. From using the primary data gathered it will become clear to see if this could be the case.

The research indicated an overwhelming majority of participants feel that technology is being forced upon their business and proceeded to list factors that fall into the initial framework [21], such as; government, external and competitive pressures to be to blame.

Some participants argued that: "we need to use it (technology) to keep up with modern day society" while another said "we are just told what to do, then go out and do it" (regarding adopting new technology).

Many felt because of these factors it is almost required to have IT systems in their business, and many feel businesses need technology for modern day society.

When further asked about modern-day society the feedback given was surprisingly surrounding the customer base of SMEs. One response was "all my customers are online, all the time. They are always on the internet. So, I thought... I need to be on the internet too". Participants interviewed with running websites mentioned the importance of being always available to the consumers. This feedback was interesting as the IT adoption framework mentions little about the consumers being a factor in developing new IT systems. Despite this factor not being considered, this would be a good area to investigate further.

In addition another participant mentioned "I was forced to update from paper-based systems to online systems, and I was not happy at the time". When discussing this new IT adoption, the idea that they had the change their whole system was not initially perceived well, as shown by the previous participant. This directly follows the suggested theory that external factors may be negatively viewed [26]. This also relates to potential problems with new technology and has forced the business to review their existing practices and create new methods of practice in line with the Internet [14]. This then takes time and effort, which many of the smaller SMEs do not wish to give.

This was not the only case of a participant mentioning they feel forced into using technology. Other participants said, "Every few years we need to go out and buy a new computer and a new computer package" and "It is getting ridiculous how many computers we have to buy". This came with the begrudging attitude that SMEs must pay the hefty costs involved in updating their systems. 
The idea that choice and perspective of adoption technology are linked shows to have some potential, however, this would need to be further investigated using larger groups and more diverse groups too, as this is one area the authors feel this research poses potential limitations.

\subsection{SMEs perceptions of current and future developments in technology}

This area builds on the concept of Celuch [22] who feels that the perception of SMEs on technology relates to the intention of adopting it. This category is to try and identify any trends surrounding SMEs perceptions of IT and the possibility of future adoption of new IT.

This area proved rather interesting after analysing the primary research. All but one of the participants claimed they were content with the current level of technology usage in their company and did not want any more. As one participant put it "not wanting any more stuff (technology) to make it more confusing". While another mentioned that "cannot afford to waste away on new technology".

However, findings were split between those who were in favour of new future developments and those wanting to stay at the level they are now.

Specifically, findings from two participants were "I am about to add a new account managing system to help me keep track of cash flows" and another said, "I will need more systems in place if the business gets any larger just to keep track of everything". Both participants had a positive attitude towards technology and helped identify a possible trend.

Those in favour of adopting new technology could be defined into one of two categories from this primary research. Generally, the owners and managers of SMEs were more positive towards the potential idea of adopting new IT, which fits with Harindranath et al. [25] and their idea that managers ultimately have the power and control do to so, therefore, they may take it as a more serious suggestion. The second of those groups were the younger of the people interviewed. The idea that young people are more technologically learned is key to this suggestion, alongside the idea of good perception and intention of adopting new IT [22].

Lastly, another participant shared "we are in the process of buying new computers to keep up with new technology". The suggestion here was this participant felt the main concern about not adopting new systems is not knowing enough about available systems. This gives off the impression that SMEs need to be better informed of the technology available to them and therefore the question might be "how can SMEs become more technologically aware?"

\subsection{SMEs perceptions on MTD}

When investigating MTD via the literature review, it was clear to see there was little existing literature specifically covering this type of a system. After applying existing theories to the concept of MTD, theories suggested that MTD might be negatively viewed by SMEs due to the nature of its introduction.

Most participants shared a similar look when discussing MTD, a look of confusion and worry. MTD will begin the month this study is completed, and the government is planning to introduce MTD to all taxpaying SMEs. When asked about MTD each SME had little knowledge of the proposed new system. Taking into consideration the release of MTD is imminent there is very little known about it, this does seem rather worrying. After gaining permission to share additional information via a letter, one self-employed accountant wrote "I am making aware the merger of Business 1 and Business 2. This will have no impact on current clients and processes". This person also disclosed in an interview, "MTD will have a huge impact on the agricultural and farming industry as they don't have computers and rely on paper and pen most of the time". This suggests there will be upset within the farming industry and implies they are being forced to adopt new technology, of which they may not want to or they might not have the skills or means to do so.

Of those interviewed similar trends emerged. Very little is still known about MTD, and many will be relying on their accountants to help them through this technological change. Furthermore, many SMEs are worried about the costs that they will incur since they might need to outsource the usage of the system to their accountants. To initiate MTD SMEs must purchase the software, then take time learning how to use the new software. Linking this to the choice and perspective concept [22], MTD is very fitting to the idea of the concept, as it has a very negative view surrounding it after being forced upon SMEs.

Some of the participants intend to try and avoid it completely which for them would mean making less money. However, SMEs value their time greater than learning to use a new system even if that system can bring them greater 
financial returns. It is noted that one participant claimed MTD would be 'a step too far' and would not be involving themselves with it.

Another trend identified regarding MTD, is that most of the negative feedback came from the older participants, therefore this suggests again that investigating age and new technology perception could be an interesting area to look at in the future.

Lastly, regarding MTD there was one participant who saw MTD in a different light and is happy about the new system as they feel "it will mean fewer errors than the current system and would help". It should be noted this feedback came from the largest sized business participant and this could justify that reason.

The authors believe that time will aid research when considering MTD, as it is a new concept and needs time to be introduced and better understood by businesses.

\subsection{Are technologies necessary for SMEs?}

The literature suggests that SMEs cannot cope without technology. In line with this, this research has been conducted as part of the semi-structured interviews in an effort in developing a deeper understanding of this area. This will be a good opportunity to develop a deeper understanding of how SMEs truly view technology, and how it plays a role in the business world.

Despite all negative comments made throughout each interview, when asked if SMEs could cope without technology, every participant believed technology to be necessary. They all felt they could not run their business without technology. From here a trend started to emerge, the main source of technology said they could not cope without was the Internet. The Internet has become a vital part of SMEs operation. One participant shared that "I would not want to go back to the old ways at all, as there was too much to sort and remember, whereas technology has helped solve this issue". It seems the use of the Internet is one of the most important technology used for SMEs.

\section{Conclusion}

This study aimed to examine the existing literature and find gaps for further research. Gaining a broad understanding of why SMEs use technologies as well as their perception on technological change gave some very interesting insights. This gave depth and understanding of a possible thought process of how SMEs would decide on technology adoption.

MTD was the most difficult area to cover as the new concept has little existing data, therefore, this might be an area worth further investigation once the new technology becomes more embedded in SMEs operations.

Overall, each area of investigation produced gaps within the research, which were known as the key areas of interest throughout this study. Our research enables academics and practitioners to gain a further insight and understanding into how or why SMEs use technologies as well as how they perceive them and are impacted by them. With further understanding of the area it became apparent that most reasons were due to self-interest rather than the interest of the business. Examples of which being long term time and cost reduction as suggested in the literature review [6]-[9].

Perceptions of current and future technology were at the heart of this study and an area of which is noted throughout. During the secondary research, the development of ideas from Ghobakhloo et al. [21] and Celuch [22] helped bring SMEs perception of technology to life. The use of secondary research helped develop a stronger idea of SMEs perception and started to show a further level of understanding through analysis and comparison of existing research. From the existing research, more development was appearing in the study. However, research conducted was not all-surrounding SMEs perception of technology and did not offer any additional information surrounding the topic, which developed a key area of interest to further investigate this via primary research.

From the primary research a much more in depth and focused understanding was developed. The primary research allowed further investigating of specific perceptions SMEs may have. Analysing the primary research helped form trends in the research surrounding perceptions such as; younger participants responding better to new technology and a common belief that SMEs cannot live without technology.

Initial research surrounding MTD was limited as it is a new concept to many. The main source of understanding 
found regarding SMEs perception was through the primary research. From this primary research, it was clear to notice most participants sharing their negative standpoints on the matter as this was something of a great concern to them. Additionally, exploration into this idea helped to develop a further understanding of MTD, as well as developing a strong insight into the perception that SMEs have.

\section{Conflict of interest}

The authors declare no conflict of interest.

\section{References}

[1] WebStats, "Internet growth statistics 1995 to 2019-the global village online," 2020. [Online]. Available: https:// www.internetworldstats.com/emarketing.htm.

[2] A. Hunsaker and E. Hargittai, “A review of Internet use among older adults," New Media \& Society, vol. 20, no. 10, pp. 3937-3954. Available: doi: 10.1177/1461444818787348.

[3] S. Mathews, C. Bianchi, K. Perks, M. Healy and R. Wickramasekera, "Internet marketing capabilities and international market growth," International Business Review, vol. 25, no. 4, pp. 820-830. Available: doi: 10.1016/ j.ibusrev.2015.10.007.

[4] C. Rhodes, Business statistics. UK: House of Commons Library, 2018.

[5] K. Hoffman, M. Parejo, J. Bessant and L. Perren, "Small firms, R\&D, technology and innovation in the UK: A literature review," Technovation, vol. 18, no. 1, pp. 39-55, 1998.

[6] M. Quayle, "E-commerce: The challenge for UK SMEs in the twenty-first century," International Journal of Operations \& Production Management, vol. 22, no. 10, pp. 1148-1161. Available: doi: 10.1108/01443570210446351.

[7] T. Latter, "The benefit of an integrated system-how our tech can help," 2017. [Online]. Available: https:// thehappinessindex.com/blog/tech/integrated-system-benefits/.

[8] M. A. Nugroho, A. Z. Susilo, M. A. Fajar and D. Rahmawati, "Exploratory study of SMEs technology adoption readiness factors," Procedia Computer Science, vol. 124, pp. 329-336, 2017.

[9] C. E. Sunday and C. C. E. Vera, "Examining information and communication technology (ICT) adoption in SMEs," Journal of Enterprise Information Management, vol. 31, no. 2, pp. 338-356, 2018.

[10] L. Li, F. Su, W. Zhang and J. Mao, "Digital transformation by SME entrepreneurs: A capability perspective," Information Systems Journal, vol. 28, no. 6, pp. 1129-1157. Available: doi: 10.1111/isj.12153.

[11] S. Alam and M. Mohammad Noor, "ICT adoption in small and medium enterprises: An empirical evidence of service sectors in Malaysia," International Journal Of Business And Management, vol. 4, no. 2, pp. 112. Available: doi: 10.5539/ijbm.v4n2p112.

[12] J. Bell and S. Loane, "New-wave global firms: Web 2.0 and SME internationalisation," Journal of Marketing Management, vol. 24, no. 3-4, pp. 213-229. Available: doi: 10.1080/02672571003594648.

[13] F. Kitsios and M. Kamariotou, "Decision support systems and strategic planning: Information technology and SMEs' performance,” International Journal of Decision Support Systems, vol. 3, no. 1-2, pp. 53-70, 2018.

[14] H. Shiels, R. McIvor and D. O’Reilly, "Understanding the implications of ICT adoption: Insights from SMEs," Logistics Information Management, vol. 16, no. 5, pp. 312-326. Available: doi: 10.1108/09576050310499318.

[15] I. Stankovska, S. Josimovski and C. Edwards, "Digital channels diminish SME barriers: The case of the UK," Economic Research-Ekonomska Istraživanja, vol. 29, no. 1, pp. 217-232. Available: doi: 10.1080/1331677x.2016.1164926.

[16] I. Papasolomou and Y. Melanthiou, "Social media: Marketing public relations' new best friend," Journal of Promotion Management, vol. 18, pp. 319-328, 2012.

[17] L. Steinhoff, D. Arli, S. Weaven and I. V. Kozlenkova, "Online relationship marketing," Journal of the Academy of Marketing Science, vol. 47, pp. 369-393, 2019.

[18] D. Skoumpopoulou and A. Robson, "Systems change in UK HEIs: How do culture, management, users and systems align," Journal of Enterprise Information Management, 2020.

[19] V. Parthasarathy and V. Kumar, "Determinants of cloud computing adoption by SMEs," International Journal of Business Information Systems, vol. 22, no. 3, pp. 375-395, 2019.

[20] J. Mehrtens, P. Cragg and A. Mills, “A model of Internet adoption by SMEs," Information \& Management, vol. 39 , no. 3, pp. 165-176. Available: doi: 10.1016/s0378-7206(01)00086-6. 
[21] M. Ghobakhloo, T. Hong, M. Sabouri and N. Zulkifli, "Strategies for successful information technology adoption in small and medium-sized enterprises," Information, vol. 3, no. 4, pp. 36-67. Available: doi: 10.3390/info3010036.

[22] K. Celuch, "SME internet use: The moderating role of normative influence," Journal of Small Business Strategy, vol. 24, no. 2, pp. 69-90, 2014.

[23] S. Baines, "New technologies and old ways of working in the home of the self-employed teleworker," New Technology, Work and Employment, vol. 17, no. 2, pp. 89-101. Available: doi: 10.1111/1468-005x.00096.

[24] M. Levy and P. Powell, "Exploring SME Internet adoption: Towards a contingent model," Electronic Markets, vol. 13, no. 2, pp. 173-181. Available: doi: 10.1080/1019678032000067163.

[25] G. Harindranath, R. Dyerson and D. Barnes, "ICT adoption and use in UK SMEs: A failure of initiatives," Electronic Journal Information Systems Evaluation, vol. 11, pp. 91-96, 2019.

[26] L. Deckers, Motivation. New York: Routledge, 2018.

[27] R. Dholakia and N. Kshetri, "Factors impacting the adoption of the Internet among SMEs," Small Business Economics, vol. 23, no. 4, pp. 311-322. Available: doi: 10.1023/b:sbej.0000032036.90353.1f.

[28] Gov.uk, "Overview of making tax digital," 2020. [Online]. Available: https://www.gov.uk/government/publications/ making-tax-digital/overview-of-making-tax-digital.

[29] A. J. Sawyer and S. James, "Digitalization of tax: Comparing New Zealand and United Kingdom approaches," Business and Law: Journal Articles, vol. 275, pp. 1-20, 2018.

[30] J. Creswell, Research design: Qualitative, quantitative, and mixed methods approaches. London: SAGE, 2014.

[31] G. Gibbs, Analyzing qualitative data. London: SAGE, 2018.

[32] M. Marshall, "Sampling for qualitative research,” Family Practice, vol.13, no. 6, pp. 522-526. Available: doi: https:// doi.org/10.1093/fampra/13.6.522.

[33] M. Miles and A. Huberman, Qualitative data analysis an expanded sourcebook. Thousand Oaks: SAGE, 1994. 